\title{
Hospital Doctors' Views and Concerns about Pharmacovigilance
}

Vallano $A^{1,2^{\star}}$, Castañeda PF${ }^{1}$, Quijada Manuitt MA ${ }^{1,2}$, Simon PC ${ }^{1}$, Pedrós $C^{1}$, Quintana B' ${ }^{1}$ Esterlich $E^{1}$, Arnau JM ${ }^{1,2}$

${ }^{1}$ Clinical Pharmacology Service, Bellvitge University Hospital, IDIBELL, L'Hospitalet de Llobregat, 08907 Barcelona, Spain

${ }^{2}$ Department of Pathology and Experimental Therapeutics, University of Barcelona, L'Hospitalet de Llobregat, 08907 Barcelona, Spain

\begin{abstract}
Purpose: The aim of the study was to evaluate the opinions and concerns of hospital doctors about adverse drug reactions (ADRs) and pharmacovigilance.
\end{abstract}

Methods: A qualitative study was undertaken using focus groups in sessions on pharmacovigilance activities conducted in thirteen clinical services of a tertiary university hospital. A total of 296 physicians participated in these sessions by giving their opinions or expressing their doubts about ADR and pharmacovigilance activities which were recorded by different observers and subsequently analysed.

Results: Doctors remarked on: a) the importance, concern, frequency and specific types of ADRs that were observed in clinical practice; b) problems of clinical decision making related to the suspected ADRs; c) methods for improving detection and reporting ADRs; d) monitoring of specific ADRs or ADRs caused by specific drugs; e) and measures to prevent and minimize the risk of ADRs. Physicians expressed doubts related to: $a$ ) the basic concepts of ADRs; b) the methods of ADR identification and evaluation; c) the objectives and procedures of pharmacovigilance programmes; d) and the impact of pharmacovigilance activities.

Conclusions: Hospital doctors believe that ADRs are a matter for concern in their daily clinical practice, and monitoring ADRs as well as measures for preventing the risk of ADRs are needed. Nevertheless, doctors have doubts about what an ADR is, the accuracy of diagnostic methods, the development of pharmacovigilance activities and their impact on clinical practice. Pharmacovigilance should be better explained through a continuous feedback and close relationship with hospital doctors.

Keywords: Adverse drug reactions; Pharmacovigilance; Doctors; Hospital

\section{Introduction}

Adverse drug reactions (ADRs) are a relevant problem in health care, and particularly in the hospital setting [1-3]. Unfortunately, ADRs are underreported in general [4], and particularly in hospitals [5]. Knowledge and attitudes of health professionals appear to be strongly associated with underreporting in a high proportion of studies [6]. Therefore, it is important to know the opinions, perceptions and attitudes of hospital doctors with respect to ADRs because their knowledge can help to improve the spontaneous reporting of ADRs. Several studies have assessed the knowledge, behaviour and attitudes of physicians regarding ADRs and their reporting to the pharmacovigilance systems [7-14]. Nevertheless, those studies have not evaluated specifically the doubts, uncertainties, misunderstandings and misconceptions that medical doctors may have about ADRs and pharmacovigilance systems, which are barriers to the development of pharmacovigilance activities. Thus, the objective of our study was to analyze the opinions and doubts that hospital doctors have about ADRs and pharmacovigilance activities.

\section{Methods}

A survey was carried out in a tertiary university hospital. The design of the study was qualitative because this type of methodological research allows us to understand the perceptions of doctors adequately. The qualitative technique used in the study was the focus group discussion methodology [15]. Participants were selected using a theoretical sampling model. Focus group discussions were carried out among physicians (house staff and residents in training) from different medical specialties. The groups were naturally occurring using pre-existing groups of people who worked together in the same medical department of the hospital. Thirteen focus group non- structured discussions were conducted and 296 physicians (each focus group had approximately 22 participants) from the following medical specialities participated: Anaesthesiology, Cardiology, Dermatology, Endocrinology, Gastroenterology, Infectious diseases, Internal medicine, Intensive care medicine, Nephrology, Pneumology, Neurology, Psychiatry, and Rheumatology.

Focus groups were held from January 2008 to December 2013, and the running of the groups took place in the annual pharmacovigilance sessions in each of the departments of the medical specialities described above. These sessions lasted from 45 to 60 minutes in which the pharmacovigilance program of the hospital was introduced. In the first part of the sessions, the global results of the program and the specific results relating to each medical department were presented by a clinical pharmacologist specialised in pharmacovigilance. News and other topics to do with pharmacovigilance were also introduced. In the second part of the sessions, the participants were encouraged to share their points of view and doubts about the different aspects of ADRs and pharmacovigilance activities, and an open discussion was held. The participants were told that the purpose of the sessions was to explain pharmacovigilance activities, as well as to understand their perception and doubts about ADRs and pharmacovigilance.

*Corresponding author: Antonio Vallano Ferraz, Clinical Pharmacology Service, Bellvitge University Hospital, Feixa Llarga s/n, 008907 L'Hospitalet de Llobregat, Tel : 93260 7221; E-mail: avallano@bellvitgehospital.cat

Received February 24, 2015; Accepted March 17, 2015; Published March 23 2015

Citation: Vallano A, Castañeda PF, Quijada Manuitt MA, Simon PC, Pedrós C et al. (2015) Hospital Doctors' Views and Concerns about Pharmacovigilance. J Pharmacovigilance 3: 160. doi:10.4172/2329-6887.1000160

Copyright: $\odot 2015$ Vallano A, et al. This is an open-access article distributed under the terms of the Creative Commons Attribution License, which permits unrestricted use, distribution, and reproduction in any medium, provided the original author and source are credited. 
A pharmacologist acted as the moderator, and encouraged the participants to discuss the topic and guided the discussion. Another clinical pharmacologist, acting as an independent observer, took notes on the subjects that emerged from the discussion in each session. These notes were contrasted with other clinical pharmacologists, who also attended the sessions. A coding method and framework analysis with the data collected was performed (transcription, familiarisation with the interview, coding, developing a working analytical framework, applying the analytical framework, charting data into the framework matrix, interpreting the data) [16].

\section{Results}

An overview of the issues identified in the sessions are shown in (Tables 1 (points of view) and 2 (doubts)).

\section{Opinions}

Impact of ADRs on clinical practice: Doctors often stated that ADRs were important in their experience from a clinical perspective in terms of concerns, frequency, and clinical consequences. For instance, one doctor said, "One situation that worried us is mandibular osteonecrosis produced by bisphosphonates because we often prescribe these drugs". In addition, doctors usually talked about characteristics of ADRs seen in their practice with particular emphasis on the drugs most used in their area of clinical expertise. For example, another doctor said, "We often see adverse effects as renal dysfunction produced by angiotensin converting enzyme (ACE) inhibitors or angiotensin II receptor antagonists and diuretics, generally in elderly patients with risk factors such as diarrhoea or dehydration". Another physician said, "We see cases of pathological gambling in patients treated with dopamine agonists". Doctors reported many other cases of ADRs, relating to a specific drug or a pharmacological group, which were identified in their clinical practice.

Problems of diagnosing ADR: Doctors also explained they frequently had problems taking clinical decisions on causation related to clinical situations with adverse events and with drugs that were suspected of causing those events. For instance, one physician revealed, "In hospitalised patients with hepatic enzymes abnormalities and thrombocytopenia, we have problems in the differential diagnosis when we suspect the implication of medicines". Another physician said, "We sometimes have difficulties to establish a causality relationship between some conditions, such as infections or progressive multifocal leukoencephalopathy, and immunosuppressive drugs". This problem was more serious when patients were treated with multiple medicines. One doctor said, "It is difficult to know whether adverse events are due to a specific drug, especially when patients are treated with multiple drugs".

1. Impact of ADRs on hospital doctors' clinical practice

2. Problems of hospital doctors in diagnosing ADRs

3. Methods for improving detection and reporting ADRs

4. Monitoring of specific ADRs produced by specific medicines

5. Measures to prevent and minimise ADRs in hospital
Measures for improving detection and reporting ADRs: Doctors also gave their personal opinions about methods to improve ADR reporting and detection in the hospital based on their own clinical experience. For example, one physician said, "ADRs in the hospital could be reported clicking on specific tabs in the electronic medical records". Alternative methods for identifying ADRs were proposed by hospital doctors. Another doctor said, "It might be useful to identify ADRs occurring in the hospital through reviewing hospital discharge reports".

Monitoring of particular ADRs produced by specific medicines: Doctors made contributions about the importance of monitoring particular ADRs produced by specific groups of medicines, especially those related to their own clinical areas or with new marketed medicines that have new mechanisms of action and following up patients treated with those drugs. For example one of them said, "Each time it is more typical to use medicines with new mechanisms of action such as medicines that inhibit TNF- $\alpha$ factor. It would be very useful to do a follow-up on these medicines especially on everything that it is related to the safety of these drugs in clinical practice".

Measures to prevent and minimize ADRs in the hospital: The doctors were concerned that very often a lot of patients, especially the elderly, are polymedicated with several drugs that they do not really need. Hence, they proposed checking these prescriptions and withdrawing the unnecessary medicines. One doctor noted, "We frequently see polymedicated patients. It would be worthwhile checking all the medicines that patients are being treated with and withdrawing those ones that the patient does not need". Doctors also suggested avoiding some drugs in patients who had risk factors for developing specific ADRs. For instance, one doctor said, "We should avoid using nonsteroidal anti-inflammatory drugs in patients with impaired renal function". Another doctor underlined the importance of using additional tests in order to prevent or reduce ADRs. For example, one doctor said, "It might be very useful to monitor plasma concentrations of antiretroviral medicines with the purpose of better adjusting the dose, and thus avoiding ADRs".

\section{Doubts}

Doubts about the concept of ADRs: Physicians still have many doubts about what ADRs are. For example, one doctor asked, "What is an ADR?" Another doctor asked, "Can we consider the effects derived from the mechanism of action of the medicine as an ADR?" Doctors also have doubts about specific aspects related to ADRs, such as the severity. For instance, one doctor asked, "What does a serious ADR mean?".

\footnotetext{
ADRs concern hospital doctors
}

ADRs are very frequent in the experience of hospital doctors

ADRs have important clinical consequences

ADRs are usually specific depending on the clinical speciality

Difficulties with clinical decisions relating to ADRs

Difficulties with patients treated with several drugs

Reporting ADRs using electronic medical records

Review of hospital discharge reports

Follow up of patients treated with specific groups of medicines

Follow up of patients treated with medicines with new mechanisms of action

Review of prescribed drugs in patients with polypharmacy

Avoiding specific groups of drugs in patients with risk factors

Testing for pharmacological parameters

Table 1: Hospital doctors' opinions on pharmacovigilance activities. 
1. Doubts related to the basic concepts of ADRs

2. Doubts about the methods of $A D R$ identification and evaluation

3. Doubts about objectives and procedures of the Pharmacovigilance programmes

4. Doubts about the impact of the Pharmacovigilance activities
Meaning of ADR

ADRs directly derived from the mechanisms of action of medicines

Severity criteria of ADRs

Methods of ADR identification

Methods of evaluation of medicine causality

Objectives of the Pharmacovigilance programmes

Operational procedures of the Pharmacovigilance programmes

Usefulness of the Pharmacovigilance activities

Effect on patient safety and the safe use of drugs

Decision-making process on the use of drugs relating to public health

Table 2: Hospital doctors' doubts about the pharmacovigilance activities.

Doubts about the methods of identification and evaluation of ADRs: Doctors had doubts about the how to identify and evaluate ADRs within the context of pharmacovigilance activities. Thus, the physicians asked several questions related to the process of identification and evaluation of ADRs, "How are ADRs identified?", "Who makes the evaluation?", "How are the cases of suspected ADRs evaluated?", "How is the casual relationship between ADRs and medicines established?". In addition, doctors were uncertain about the true causal relationship between adverse events and several groups of drugs or specific drugs, which have recently been reported on in medical journals.

Doubts about the objectives, and procedures of pharmacovigilance programmes: The participants frequently asked questions about the pharmacovigilance programmes because they did not know how our hospital pharmacovigilance programme and the Spanish System of Pharmacovigilance work. For example, they asked several questions such as, "Who has to send the yellow cards when ADRs are suspected?", "Where are these yellow cards to be sent?", "Should all ADRs be reported, even though they are very well-known ADRs?", "Should cases of ADRs in patients treated with multiple medicines be reported, when it is very difficult to know what the causative medicine is?", "What is the procedure to identify diagnoses related to ADRs in the hospital?", "How do you establish a causal link between a drug and an adverse event?"

Doubts about the impact of pharmacovigilance activities: Clinicians had doubts about the usefulness and impact of pharmacovigilance activities on patients' safety. The doctors had several doubts about the decision-making process relating to drug use and public health. Thus, some clinicians asked several questions on these topics, such as "Does the information in cases of suspected ADRs go on the drug information sheet?", "How is the information added or changed on safety on the drug data sheet?, "How are decisions taken when a medicine is withdrawn from the pharmaceutical market?", "How useful are the warnings on drug safety?", "How are the warnings about drug safety followed up on?", "How could pharmacovigilance activities prevent ADRs?.

\section{Discussion}

Our study has identified the key opinions and doubts that hospital physicians have on issues relating to ADRs and pharmacovigilance, which are relevant to the development of pharmacovigilance activities. It should be noted that pharmacovigilance activities in hospitals identify serious ADRs resulting in hospitalisation, and there are ADRs occurring in hospitalised patients, often with a high comorbidity, after they have received drugs that are only administered in this setting [17]. It is noteworthy that analysing the views and doubts of hospital physicians is important because they are less likely to report ADRs [5].

The results of this study show that hospital physicians have positive opinions and attitudes about pharmacovigilance, and they usually consider that pharmacovigilance activities and the reporting of ADRs are an important part of good clinical practice. This attitude is probably related to the perception of the relevant impact of ADRs on clinical practice. However, in spite of this fact several studies have shown unsatisfactory levels of knowledge of ADRs among hospital doctors [18-21]. Different studies consistently indicate there is still an underreporting culture of ADRs among clinical practitioners [4]. Moreover, accumulating evidence suggests that doctors' attitudes to their national pharmacovigilance schemes significantly influence reporting rates and attitudes vary substantially between countries [9]. Variations can be explained by looking at economic and social factors. For example, high income countries have the highest ADR reporting rates and low income countries the lowest, even though large variations across countries in each group have also been reported [22]. Other studies have also assessed the opinions, attitudes and knowledge of pharmacovigilance among other health professions like nurses and pharmacists in hospitals $[23,24]$. These health professionals also have a very positive opinion about pharmacovigilance activities, as well as optimal attitudes, but they also underreport ADRs.

This study also focused on evaluating doubts clinicians had. Doctors asked different types of questions about pharmacovigilance activities, as well as about several aspects relating to ADRs that they usually encountered in their own clinical practice. The doubts and misconceptions doctors had about ADRs and pharmacovigilance activities could play an important role in explaining the underreporting of ADRs. It is important to note that, surprisingly, physicians' doubts and misconceptions on the meaning of ADR were revealed. There are doctors who believe that the effects related to the mechanism of action of drugs are not ADRs, and only unexpected effects are ADRs. This finding is extremely important because the interpretation of the term $\mathrm{ADR}$ is a key factor influencing the results of surveys on ADRs [17].

Hospital doctors also expressed doubts on accurate methods for diagnosing ADRs. Previous studies have already reported on the misconception that absolute confidence in the diagnosis of an ADR is important in deciding whether to report a case [8]. Differential diagnosis of ADRs is a difficult and complex process because for each adverse event there are many possible causes either pharmacological or non-pharmacological. Hence, doctors assessing ADRs face the need to make causality assessment judgements on drug-related events. Several methods for assessing causality of ADRs have been proposed that can be classified into three broad categories: 1) expert judgement, also called global introspection; 2) algorithms; 3 ) and probabilistic methods such as Bayesian approaches [25-30]. However, as a result of problems of validity and reproducibility, none of the diagnostic methods are completely satisfactory. There is still no method universally accepted for causality assessment of ADRs. Obviously, there is a need to find 
better procedures to assess ADRs. Doctors also raised questions and doubts about national and international systems of reporting ADRs, as well as the final impact and effects of pharmacovigilance activities. Several studies have also shown that doctors are not aware of pharmacovigilance activities and the national system of reporting ADRs $[12,19,20,31]$. It is very important to resolve these doubts because the involvement and participation of doctors in pharmacovigilance activities may be negatively affected if the goal and the real impact of these activities in their clinical practice are unclear to them.

There are different reasons for these doubts and lack of adequate knowledge about ADRs and pharmacovigilance activities, such as insufficient teaching of pharmacovigilance in the training of undergraduate and graduate students, and lack of updating courses on pharmacovigilance for health professionals. Education and training are the most recognised means of improving ADR reporting [18, 32]. Continuous medical education, training and integration of ADR reporting into the clinical activities of doctors would almost certainly improve reporting. Pharmacovigilance sessions can be developed and integrated into clinical activities in the hospital. The sessions can be informative and educational, as well as producing feedback and accountability for pharmacovigilance activities [33,34]. Sessions and other activities that clinicians are able to participate in can be very useful in helping to resolve these doubts and misunderstandings. In addition, feedback on pharmacovigilance activities is well received and appreciated by general practitioners and specialist doctors [35]. Doctors may need help with diagnosing ADRs, or in the process of recording and reporting ADRs, or obtaining information about pharmacovigilance alerts issued by regulatory medicines agencies, or any additional information on ADRs. Clinical pharmacologists and other pharmacovigilance experts can provide support and assistance to physicians in all these areas.

It is worth stressing that the doctors came up with some interesting ideas to enhance and encourage the reporting of ADRs, such as using the computing resources within the electronic medical records. Several studies have shown that implementing simple strategies such as increasing the availability of more yellow cards in health centres, including a hyperlink to an online ADR reporting form in hospitals' electronic patientrecords, regularvisitsbya clinical expert, or educational measures as well as small financial incentives can increase the reporting of ADRs [36-42]. ADRs are still discovered by spontaneous reporting systems in hospitals, but electronic hospital information systems and the implementation of pharmacoepidemiological approaches increase the possibilities and the value of ADR detection in hospitals [17]. Currently, new powerful and innovative computer systems to detect ADRs, based on exploiting clinical data from electronic health care records using biomedical computer technologies, are in development, which may provide new opportunities for the future development of pharmacovigilance activities supplementing the spontaneous reporting system $[43,44]$. In the future, more studies are needed to assess the impact of different measures and strategies in order to improve the quantity and quality of reporting ADRs. Moreover, doctors made proposals to reduce ADRs, such as reviewing drug treatments, and withdrawing unnecessary medication. Computerized surveillance has proved to be a useful technique in order to understand and prevent ADRs, as is the assistance of experts in the drug prescribing process [4548]. However, the quality of the implementation process is dependent on the physician using the software correctly [49].

Our study has several limitations. The methodology of the study was qualitative, and we did not use a structured questionnaire in order to collect information in the form of quantitative data. Therefore, we did not quantify the number of responses. However, in qualitative studies, the content of information (that is expressed in the form of sentences that reflects opinions and ideas) is more important than the amount of information referred to as a number of responses. It was a study carried out in a single centre, albeit a high technology tertiary hospital, where we gathered information from doctors representing various medical specialities. However, information from other medical specialties, and other health professionals like nurses or pharmacists was not collected. As well as this, we did not identify or analyse what is happening in the primary care facilities. Thus, our results are particularly applicable to our institution, but may not apply to other institutions or to primary health care. Future studies should look at other areas and centres.

In conclusion, our study shows that doctors are concerned about ADRs, because they believe that ADRs are frequent and relevant to their daily clinical practice. There are some doubts about what an ADR is, the accuracy of diagnostic methods, the development of pharmacovigilance activities and their impact on clinical practice. These doubts could be an obstacle to developing pharmacovigilance activities in clinical practice. Training in pharmacovigilance and disseminating results from pharmacovigilance activities should be developed through a closer relationship with hospital doctors, using continuous feedback and supporting pharmacovigilance activities.

\section{Acknowledgements}

We thank Angus Powles for reviewing the English

Declaration of interest

The authors state no conflict of interest and have received no payment in preparation of this manuscript

Ethical standards

This manuscript does not contain clinical studies or patient data.

Conflict of interest

The authors declare that they have no conflict of interest.

\section{References}

1. Classen DC, Pestotnik SL, Evans RS, Lloyd JF, Burke JP (1997) Adverse drug events in hospitalized patients. Excess length of stay, extra costs, and attributable mortality. JAMA 277: 301-306.

2. Lazarou J, Pomeranz BH, Corey PN (1998) Incidence of adverse drug reactions in hospitalized patients: a meta-analysis of prospective studies. JAMA 279 1200-1205.

3. Pedrós C, Quintana B, Rebolledo M, Porta N, Vallano A, et al. (2014) Prevalence, risk factors and main features of adverse drug reactions leading to hospital admission. Eur J Clin Pharmacol 70: 361-367.

4. Hazell L, Shakir SA (2006) Under-reporting of adverse drug reactions : a systematic review. Drug Saf 29: 385-396.

5. Herdeiro MT, Figueiras A, Polónia J, Gestal-Otero JJ (2005) Physicians' attitudes and adverse drug reaction reporting : a case-control study in Portugal. Drug Saf 28: 825-833.

6. Lopez-Gonzalez E, Herdeiro MT, Figueiras A (2009) Determinants of underreporting of adverse drug reactions: a systematic review. Drug Saf 32: 19-31.

7. Bateman DN, Sanders GL, Rawlins MD (1992) Attitudes to adverse drug reaction reporting in the Northern Region. Br J Clin Pharmacol 34: 421-426.

8. Belton KJ, Lewis SC, Payne S, Rawlins MD, Wood SM (1995) Attitudina survey of adverse drug reaction reporting by medical practitioners in the United Kingdom. Br J Clin Pharmacol 39: 223-226.

9. Belton KJ (1997) Attitude survey of adverse drug-reaction reporting by health care professionals across the European Union. The European Pharmacovigilance Research Group. Eur J Clin Pharmacol 52: 423-427. 
Citation: Vallano A, Castañeda PF, Quijada Manuitt MA, Simon PC, Pedrós C, et al. (2015) Hospital Doctors' Views and Concerns about Pharmacovigilance. J Pharmacovigilance 3: 160. doi:10.4172/2329-6887.1000160

10. Eland IA, Belton KJ, van Grootheest AC, Meiners AP, Rawlins MD, et al. (1999) Attitudinal survey of voluntary reporting of adverse drug reactions. $\mathrm{Br} \mathrm{J}$ Clin Pharmacol 48: 623-627.

11. Bäckström M, Mjörndal T, Dahlqvist R, Nordkvist-Olsson T (2000) Attitudes to reporting adverse drug reactions in northern Sweden. Eur $\mathrm{J}$ Clin Pharmacol 56: 729-732.

12. Hasford J, Goettler M, Munter KH, Müller-Oerlinghausen B (2002) Physicians knowledge and attitudes regarding the spontaneous reporting system for adverse drug reactions. J Clin Epidemiol 55: 945-950.

13. Vallano A, Cereza G, Pedròs C, Agustí A, Danés I, et al. (2005) Obstacles and solutions for spontaneous reporting of adverse drug reactions in the hospital. $\mathrm{Br} \mathrm{J}$ Clin Pharmacol 60: 653-658.

14. Ekman E, Bäckström $M$ (2009) Attitudes among hospital physicians to the reporting of adverse drug reactions in Sweden. Eur J Clin Pharmacol 65: 43-46.

15. Kitzinger J (1995) Qualitative research. Introducing focus groups. BMJ 311: 299-302.

16. Gale NK, Heath G, Cameron E, Rashid S, Redwood S (2013) Using the framework method for the analysis of qualitative data in multi-disciplinary health research. BMC Med Res Methodol 13: 117.

17. Thürmann PA (2001) Methods and systems to detect adverse drug reactions in hospitals. Drug Saf 24: 961-968.

18. Oshikoya KA, Awobusuyi JO (2009) Perceptions of doctors to adverse drug reaction reporting in a teaching hospital in Lagos, Nigeria. BMC Clin Pharmaco 9: 14.

19. Chopra D, Wardhan N, Rehan HS (2011) Knowledge, attitude and practices associated with adverse drug reaction reporting amongst doctors in a teaching hospital. Int J Risk Saf Med 23: 227-232.

20. Pimpalkhute SA, Jaiswal KM, Sontakke SD, Bajait CS, Gaikwad A (2012) Evaluation of awareness about pharmacovigilance and adverse drug reaction monitoring in resident doctors of a tertiary care teaching hospital. Indian $\mathrm{J} \mathrm{Med}$ Sci 66: 55-61.

21. Khan SA, Goyal C, Chandel N, Rafi M (2013) Knowledge, attitudes, and practice of doctors to adverse drug reaction reporting in a teaching hospital in India: An observational study. J Nat Sci Biol Med 4: 191-196.

22. Aagaard L, Strandell J, Melskens L, Petersen PS, Holme Hansen E (2012) Global patterns of adverse drug reactions over a decade: analyses of spontaneous reports to VigiBase ${ }^{\mathrm{TM}}$. Drug Saf 35: 1171-1182.

23. John LJ, Arifulla M, Cheriathu JJ, Sreedharan J (2012) Reporting of adverse drug reactions: an exploratory study among nurses in a teaching hospital, Ajman, United Arab Emirates. Daru 20: 44.

24. Su CH, JiH, SuY (2010) Hospital pharmacists' knowledge and opinions regarding adverse drug reaction reporting in Northern China. Pharmacoepidemiol Drug Saf 19: $217-222$

25. Naranjo CA (1986) A clinical pharmacologic perspective on the detection and assessment of adverse drug reactions. Drug Inf J 20: 387-393.

26. Agbabiaka TB, Savović J, Ernst E (2008) Methods for causality assessment of adverse drug reactions: a systematic review. Drug Saf 31: 21-37.

27. Arimone $Y$, Bégaud $B$, Miremont-Salamé G, Fourrier-Réglat $A$, Moore N, et al (2005) Agreement of expert judgment in causality assessment of adverse drug reactions. Eur J Clin Pharmacol 61: 169-173.

28. Naranjo CA, Busto U, Sellers EM, Sandor P, Ruiz I, et al. (1981) A method for estimating the probability of adverse drug reactions. Clin Pharmacol Ther 30 : 239-245

29. Macedo AF, Marques FB, Ribeiro CF (2006) Can decisional algorithms replace global introspection in the individual causality assessment of spontaneously reported ADRs? Drug Saf 29: 697-702.

30. Lanctôt KL, Naranjo CA (1994) Computer-assisted evaluation of adverse events using a Bayesian approach. J Clin Pharmacol 34: 142-147.

31. Baraldi C, Restini A, Toda CM, Andre JN (2015) User's profile of the adverse drug reaction reporting system in Brazil: a pilot study AJPHR 113-120.

32. Santosh KC, Tragulpiankit P, Gorsanan S, Edwards IR (2013) Attitudes among healthcare professionals to the reporting of adverse drug reactions in Nepal. BMC Pharmacol Toxicol 14: 16.
33. Vallano A, Pedrós C Agustí A, Cereza G, Danés I, et al. (2010) Educational sessions in pharmacovigilance: What do the doctors think? BMC Res Notes 3: 311.

34. Sanghavi DR, Dhande PP, Pandit VA (2013) Perception of pharmacovigilance among doctors in a tertiary care hospital: influence of an interventional lecture. Int J Risk Saf Med 25: 197-204.

35. Cornelissen L, van Puijenbroek E, van Grootheest K (2008) Expectations of general practitioners and specialist doctors regarding the feedback received after reporting an adverse drug reaction. Pharmacoepidemiol Drug Saf 17: 76 81.

36. McGettigan P, Golden J, Conroy RM, Arthur N, Feely J (1997) Reporting of adverse drug reactions by hospital doctors and the response to intervention. $\mathrm{Br}$ $\mathrm{J}$ Clin Pharmacol 44: 98-100.

37. Castel JM, Figueras A, Pedrós C, Laporte JR, Capellà D (2003) Stimulating adverse drug reaction reporting: effect of a drug safety bulletin and of including yellow cards in prescription pads. Drug Saf 26: 1049-1055.

38. Figueiras A, Herdeiro MT, Polónia J, Gestal-Otero JJ (2006) An educationa intervention to improve physician reporting of adverse drug reactions: a clusterrandomized controlled trial. JAMA 296: 1086-1093.

39. Bäckström M, Mjörndal T (2006) A small economic inducement to stimulate increased reporting of adverse drug reactions--a way of dealing with an old problem? Eur J Clin Pharmacol 62: 381-385.

40. Ribeiro-Vaz I, Santos C, da Costa-Pereira A, Cruz-Correia R (2012) Promoting spontaneous adverse drug reaction reporting in hospitals using a hyperlink to the online reporting form: an ecological study in Portugal. Drug Saf 35: $387-$ 394

41. Pedrós C, Vallano A, Cereza G, Mendoza-Aran G, Agustí A, et al. (2009) An intervention to improve spontaneous adverse drug reaction reporting by hospital physicians: a time series analysis in Spain. Drug Saf 32: 77-83.

42. Gony M, Badie K, Sommet A, Jacquot J, Baudrin D, et al. (2010) Improving adverse drug reaction reporting in hospitals: results of the French Pharmacovigilance in Midi-Pyrénées region (PharmacoMIP) network 2-year pilot study. Drug Saf 33: 409-416.

43. Oliveira JL, Lopes P, Nunes T, Campos D, Boyer S, et al. (2013) The EU-ADR Web Platform: delivering advanced pharmacovigilance tools. Pharmacoepidemiol Drug Saf 22: 459-467.

44. LePendu P, Iyer SV, Bauer-Mehren A, Harpaz R, Mortensen JM, et al. (2013) Pharmacovigilance using clinical notes. Clin Pharmacol Ther 93: 547-555.

45. Kaushal R, Shojania KG, Bates DW (2003) Effects of computerized physician order entry and clinical decision support systems on medication safety: a systematic review. Arch Intern Med 163: 1409-1416.

46. Bobb A, Gleason K, Husch M, Feinglass J, Yarnold PR, et al. (2004) The epidemiology of prescribing errors: the potential impact of computerized prescriber order entry. Arch Intern Med 164: 785-792.

47. Mahoney CD, Berard-Collins CM, Coleman R, Amaral JF, Cotter CM (2007) Effects of an integrated clinical information system on medication safety in a multi-hospital setting. Am J Health Syst Pharm 64: 1969-77.

48. Ojeleye O, Avery A, Gupta V, Boyd M (2013) The evidence for the effectiveness of safety alerts in electronic patient medication record systems at the point of pharmacy order entry: a systematic review. BMC Med Inform Decis Mak 13: 69

49. van Rosse F, Maat B, Rademaker CM, van Vught AJ, Egberts AC, et al. (2009) The effect of computerized physician order entry on medication prescription errors and clinical outcome in pediatric and intensive care: a systematic review. Pediatrics 123: 1184-1190. 